\title{
Alignment and phylogenetic analysis of type II DNA topoisomerases
}

\author{
K MADHUSUDAN and V NAGARAJA* \\ Centre for Genetic Engineering, Indian Institute of Science, Bangalore 560012, India
}

MS received 6 February 1996; revised 14 August 1996

\begin{abstract}
DNA topoisomerases have been evolved to solve the topological problems of DNA during replication, transcription, recombination and segregation. Discovery of several new enzymes and their characterization has necessitated this compilation. This analysis shows the distinct evolutionary relatedness of type II DNA topoisomerases. A striking feature is the absence of a contiguous stretch of about $\mathbf{1 6 0}$ amino acids in one of the subunits of prokaryotic type II enzymes, which might have important implications to their structure and function.
\end{abstract}

Keywords. Type II DNA topoisomerases; DNA gyrase; multiple alignment; phylogenetic trees.

\section{Introduction}

DNA topoisomerases catalyse topological interconversions: supercoiling-relaxation, catenation-decatenation and knotting-unknotting of DNA. These topological events occur during important cellular processes such as replication, transcription, recombination and chromosome segregation. Thus, the enzymes are essential for the cell survival, and hence are ubiquitous. The topoisomerases are classified into two distinct subclasses based on the mechanistics of the reaction (Wang 1985; Maxwell and Gellert 1986). The type I topoisomerases break one strand of DNA and pass the other stand through the nick created and change the linking number in steps of one. On the other hand, type II enzymes cleave both stands of DNA and pass the duplex through the 'DNA gate' resulting in the change of linking number in steps of two. All known topoisomerases form a transient covalent intermediate with DNA through a phosphotyrosine linkage and reseal after strand passage. Both prokaryotes and eukaryotes have been shown to possess multiple topoisomerases, possibly evolved to provide division of labour and in certain cases as backup strategies to take care of important cellular functions. The bacterium, Escherichia coli contains two type II topoisomerases besides two type I enzymes; the yeast, Saccharomyces cerevisiae has two type I activities (Wallis et al 1989), and in humans, two isozymes of topoisomerase II have been reported (Jenkins et al 1992). Amongst all type II topoisomerases, only DNA gyrase has the ability to introduce negative supercoils into DNA in an ATP driven reaction (Gellert et al 1976). The heteromeric enzyme has been the subject of extensive study (Reece and Maxwell 1991). The second bacterial type II enzyme, topoisomerase IV, has strong decatenation and weak relaxation activities. Both the bacterial type II topoisomerases have a similar architecture and also, share considerable sequence similarity.

*Corresponding author (Fax, 91-80-3341683; E-mail, vraj@cge.iisc.ernet.in). 
In this compilation, we have focused our attention on type II topoisomerases. This is due to the wealth of information available on these enzymes, their indispensability and the degree of conservation amongst the genes from variety of organisms. On the other hand, type I topoisomerases are less conserved and only few genes are characterized. Since an elaborate discussion of sequence conservation of topoisomerases has earlier been presented (Caron and Wang 1993), we have emphasized on the evolutionary relationship.

\section{Sequence alignment and generation of evolutionary trees}

The topoisomerase II genes have been characterized from several bacteria, yeast, protozoans and higher animals. Table 1 summarizes the source and the length of the derived polypeptides. The polypeptide sequences were aligned by MACAW ver 2.0.3 using BLOSSUM62 (Schuler et al 1991; Lawrence et al 1993). This software allows manual editing of the alignment. Also, the GyrB and GyrA polypeptide sequences were fused manually and aligned with eukaryotic type II topoisomerases using Multalin (Corpet 1988). The multiply aligned sequences were subjected to PHYLIP analysis (Felsenstein 1989). The distance matrix was generated by PROTDIST of PHYLIP employing Kimura-2 parameter. The output was then analysed by NEIGHBOR applying Neighbor-joining method. The UPGMA method was used for NEIGHBOR analysis of gyrase and eukaryotic topoisomerase II alignment. The unrooted trees were generated using DRAWGRAM and DRAWTREE.

\section{Results and discussion}

This compilation and alignment of type II topoisomerases is an attempt to compile complete sequences, identify subclasses and determine the extent of phylogenetic relationships. Sequence information on DNA gyrase and eukaryotic type II topoisomerase genes has been accumulating in the databank. These reports show conservation of amino acid sequence in gyrase and also its partial homology with eukaryotic type II topoisomerases. Hence, we have presented the alignment of all deduced polypeptide sequences of type II topoisomerases in figures 1 and 2. In order to avoid errors in alignment and phylogeny analyses, we have omitted partial sequences. The alignment of A subunits of gyrase and topoisomerase IV, given in figure 1A, shows the high sequence conservation predominantly in the amino terminal region. The DNA breakage-reunion site of subunit A has the sequence AAMRYTE common to all the members. The residue Tyr-122 of E. coli GyrA, present in this sequence gets covalently attached to DNA through phospodiester bond. On the other hand, the C-terminal region does not show such extensive conservation. The dot matrix analysis, however, showed repeated sequences within this region in all GyrA sequences (Madhusudan and Nagaraja 1995). The C-terminal $33 \mathrm{kDa}$ domain of E. coli GyrA has been shown to bind DNA (Reece and Maxwell 1991).

The subunit B of bacterial type II topoisomerases shows identical patches of amino acids scattered through out the sequence. The $\mathrm{N}$-terminal $43 \mathrm{kDa}$ fragment of $E$. coli GyrB is known to retain ATPase activity, a characteristic of all type II topoisomerases. The crystal structure of this domain complexed with ADPNP has revealed the direct interaction between the protein and the cofactor (Wigely et al 1991). These contact 
Table 1. Polypeptide sequences of type II DNA topoisomerases.

\begin{tabular}{|c|c|c|c|c|}
\hline Protein & Acronym & length & $\begin{array}{c}\text { SwissProt/ } \\
\text { *EmBL } \\
\text { Acc. no. }\end{array}$ & Reference \\
\hline \multicolumn{5}{|l|}{ GyrA } \\
\hline Escherichia coli & $\mathrm{EcoA}$ & 875 & P09097 & Swanberg and Wang 1987 \\
\hline Klebsiella pneumoniae & KpnA & 876 & P14829 & Dimri and Das 1990 \\
\hline Campylobacter jejuni & CajA & 863 & Q03470 & Wang et al 1993 \\
\hline Rickettsia prowazekii & RprA & 905 & P41080 & Wood and Waite 1994 \\
\hline Bacillus subtilis & BsuA & 821 & P05653 & Moriya et al 1985 \\
\hline Staphylococcus aureus & SauA & 889 & P20831 & Margerrison et al 1992 \\
\hline Streptomyces coelicolor & ScoA & 864 & P35885 & Calcutt 1994 \\
\hline Haloferax Aa2.2 & HalA & 858 & *X60178 & Holmes and Dyall-Smith 1994 \\
\hline Mycobacterium tuberculosis & MtbA & 838 & ${ }^{*} \mathrm{~L} 27512$ & Takiff et al 1994 \\
\hline Mycobacterium smegmatis & MsmA & 854 & ${ }^{*} \mathrm{X} 84077$ & Madhusudan and Nagaraja 1995 \\
\hline \multicolumn{5}{|l|}{ GyrB } \\
\hline Escherichia coli & EcoB & 803 & P06982 & Yamagishi et al 1986; Adachi et al 1987 \\
\hline Neisseria gonorrhoeae & NgoB & 781 & P22118 & Stein et al 1991 \\
\hline Pseudomonas putida & PpuB & 806 & P13364 & Parales and Harwood 1990 \\
\hline Bacillus subtilis & BsuB & 638 & $\mathrm{P} 05652$ & Moriya et al 1985 \\
\hline Staphylococcus aureus & SauB & 640 & P20832 & Margerrison et al 1992 \\
\hline Haloferax sps. & HalB & 639 & $\mathrm{P} 21558$ & Holmes and Dyall-Smith 1991 \\
\hline Mycoplasma pneumoniae & MpnB & 650 & P22447 & Colman et al 1990 \\
\hline Spiroplasma citri & SciB & 640 & P34031 & - \\
\hline $\begin{array}{l}\text { Streptomyces sphaeroides } \\
\text { (novobiocin resistant) }\end{array}$ & $\mathrm{SspBr}$ & 677 & $* Z 17304$ & Thiara and Cundliffe 1993 \\
\hline $\begin{array}{l}\text { Streptomyces sphaeroides } \\
\text { (novobiocin sensitive) }\end{array}$ & SspBs & 684 & $* Z 17305$ & Thiara and Cundliffe 1993 \\
\hline Streptomyces coelicolor & $\mathrm{ScoB}$ & 676 & P35886 & Calcutt 1994 \\
\hline Mycobacterium tuberculosis & MtbB & 675 & *X78888 & Madhusudan et al 1994 \\
\hline Mycobacterium smegmatis & MsmB & 675 & *X84077 & Madhusudan and Nagaraja 1995 \\
\hline \multicolumn{5}{|l|}{ ParC } \\
\hline Escherichia coli & EcoC & 752 & P20082 & Kato et al 1990 \\
\hline Salmonella typhimurium & StyC & 752 & P26973 & Luttinger et al 1991 \\
\hline Staphycoccus aureus & SauE & 800 & *L25288 & Ferrero et al 1994 \\
\hline \multicolumn{5}{|l|}{ ParE } \\
\hline Escherichia coli & $\mathrm{EcoE}$ & 630 & P20083 & Kato et al 1990 \\
\hline Salmonella typhimurium & StyE & 630 & P31598 & Springer and Schmid 1991 \\
\hline Staphycoccus aureus & SauE & 663 & ${ }^{*} \mathrm{~L} 25288$ & Ferrero et al 1994 \\
\hline \multicolumn{5}{|l|}{ Eukaryotic topoisomerase II } \\
\hline ASF Virus (BA71V) & Top2_AS & 1192 & Q00942 & Garcia-Beato et al 1992 \\
\hline ASF Virus (Malawi) & Top2_AF & 1191 & P34203 & Baylis et al 1992 \\
\hline Crithidia fasciculata & Top2_CR & 1239 & P27570 & Pasion et al 1992 \\
\hline Trypanosoma brucei & Top2_TR & 1221 & P12531 & Strauss and Wang 1990 \\
\hline Trypanosoma cruzi & Top2_TY & 1232 & P30190 & Fragoso and Goldberg 1992 \\
\hline Caenorhabditis elegans & Top2_CA & 1198 & P34534 & Wilson et al 1994 \\
\hline Homo sapiens (A) & TopA_HU & 1530 & P11388 & Tsai-Pflugfelder et al 1988 \\
\hline Homo sapiens (B) & TopB $\_$HU & 1626 & QO2880 & Jenkins et al 1992 \\
\hline Mus musculus (Mouse) & Top2_MO & 1528 & Q01320 & Adachi et al 1992 \\
\hline Drosophila melanogaster & Top2_DR & 1447 & P15348 & Wykoff et al 1989 \\
\hline Plasmodium falciparum & Top2_PL & 1398 & P41001 & Ridley and Kilbey 1994 \\
\hline Saccharomyces cerevisiae & Top2_YE & 1429 & P06786 & Giaever et al 1986 \\
\hline Schizosaccharomyces pombe & Top2_SC & 1431 & P08906 & Uemura et al 1986 \\
\hline
\end{tabular}




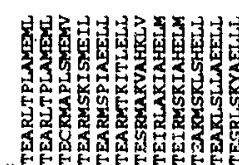

- Ect

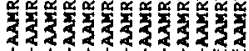

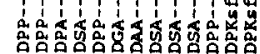

s.s.

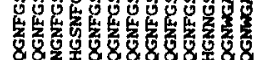

.

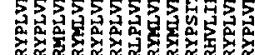

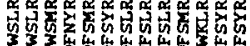

o

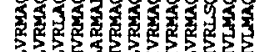

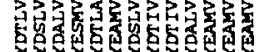

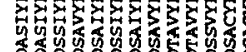

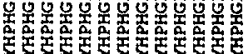

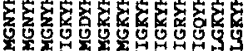

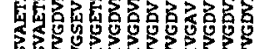

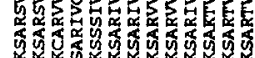

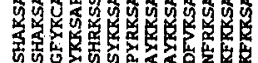

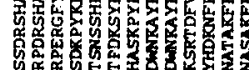

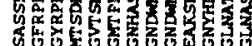

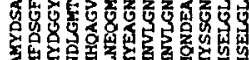

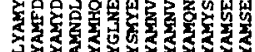

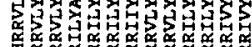

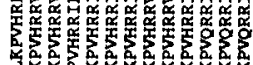

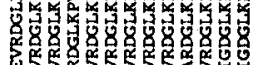

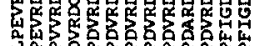

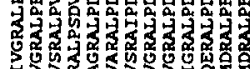

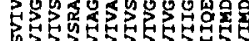

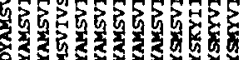

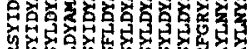

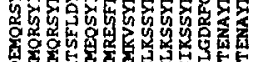

8

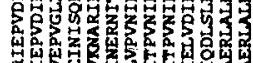

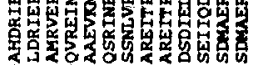

10
0

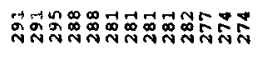

ำสำสำรัง

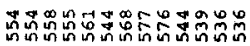

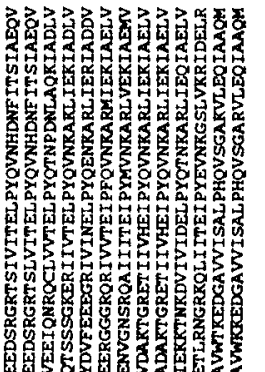

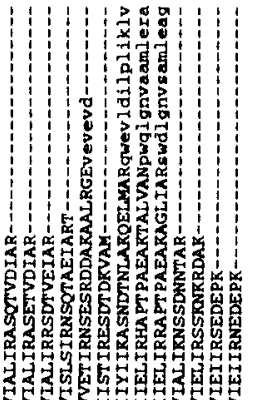

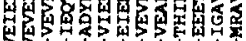

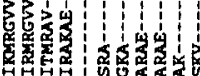

4.

U.

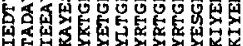

o

은

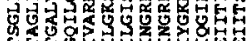

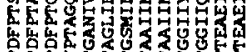

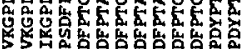

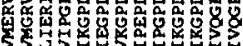

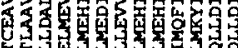

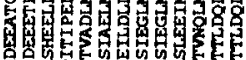

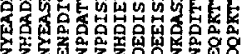

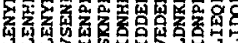

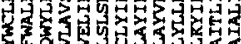

5)

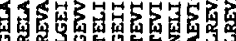

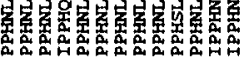

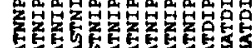

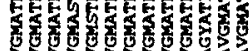

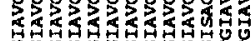

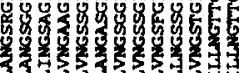

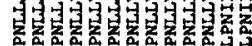

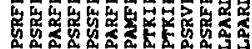

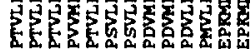

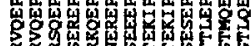

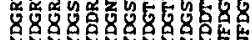

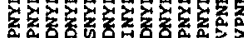

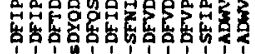

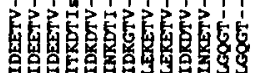

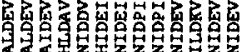

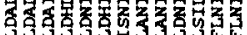

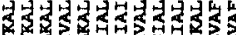

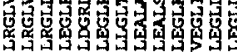

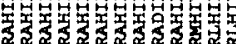

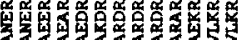

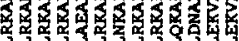

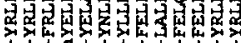

\% E E

s.

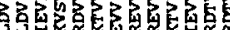

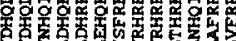

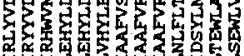

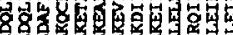

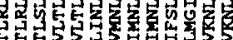

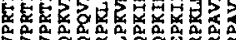

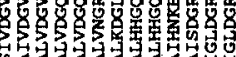

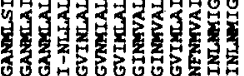

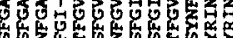

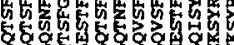

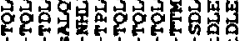

t5 d d

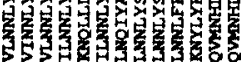

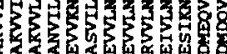

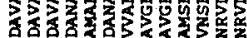

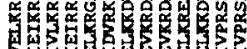

年5

年

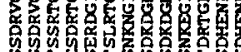

8.

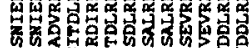

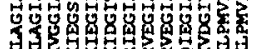

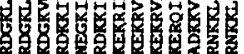

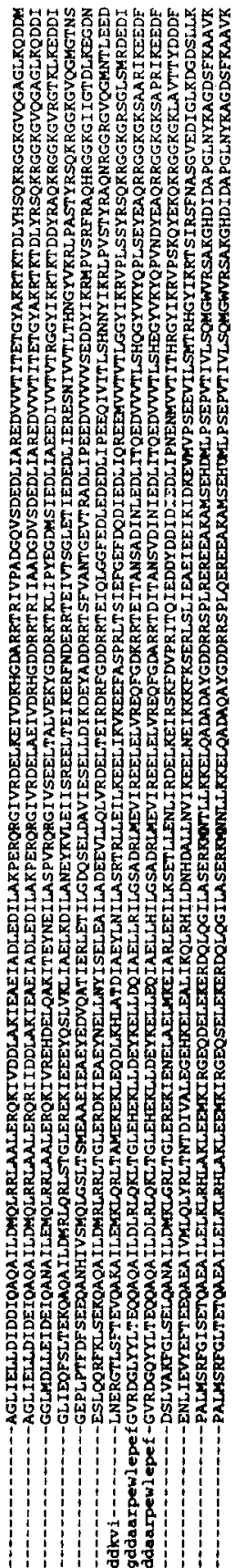

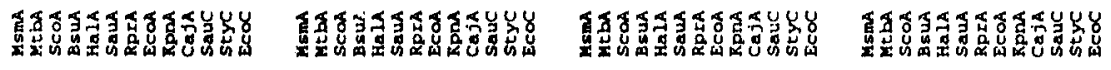




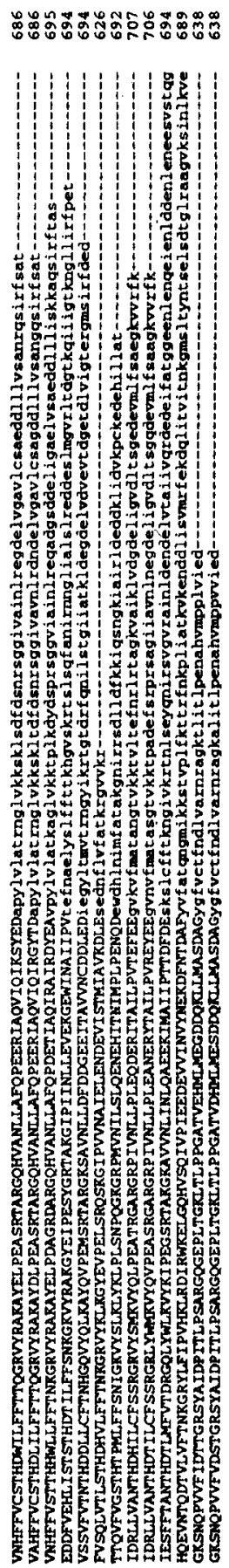

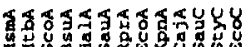

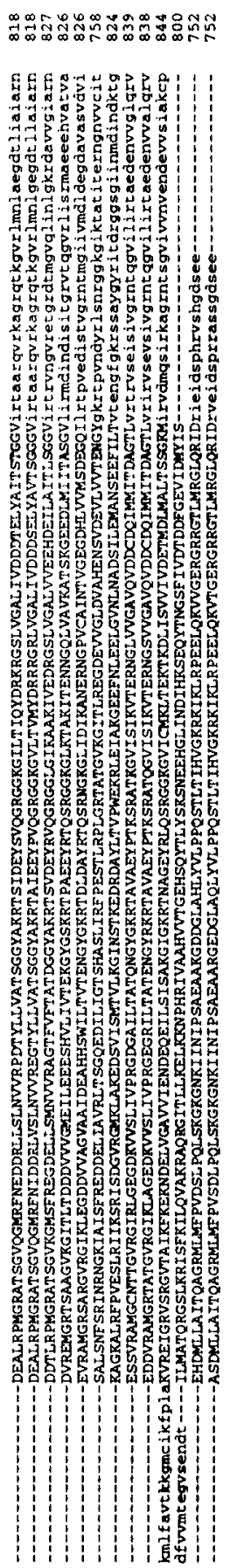

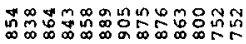

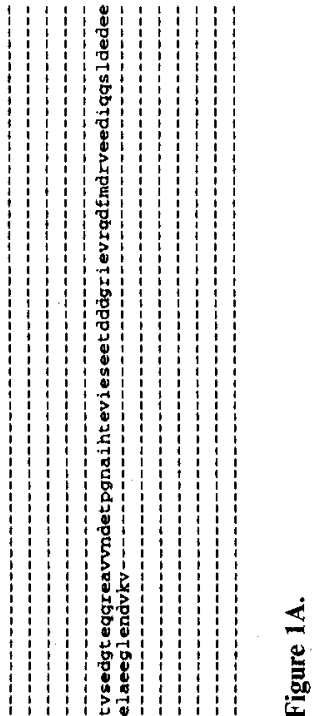

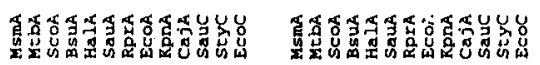




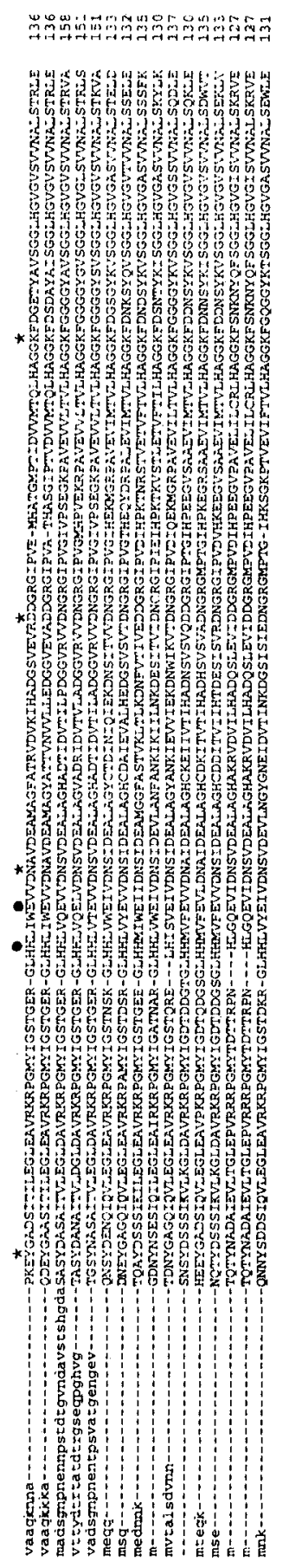

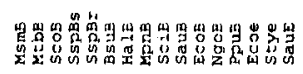

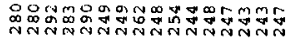

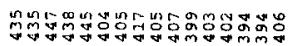

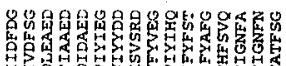
(1)

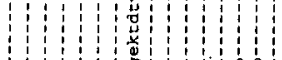

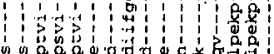

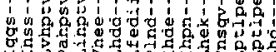

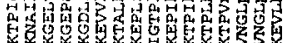

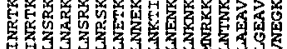

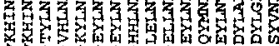
क्ष 1410 :

s:

s.

$\frac{5}{3}$

o

s. on

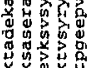

\section{.}

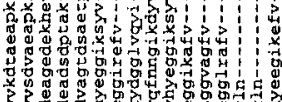

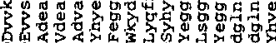

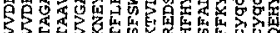

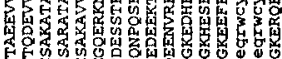

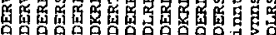

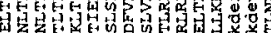

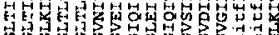

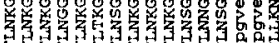

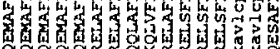

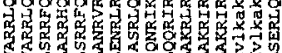

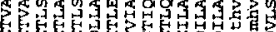

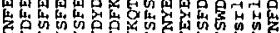

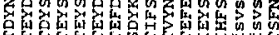

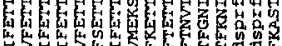

w⿰氵工力口,

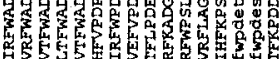

$46 \mathrm{E}_{\mathrm{E}} \mathrm{E}$

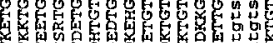

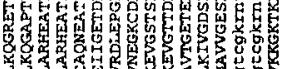

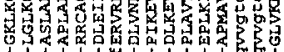

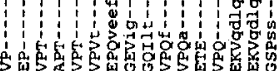

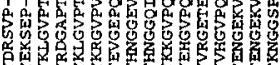

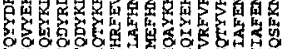

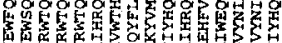

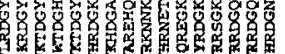

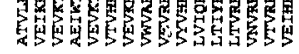

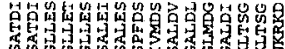

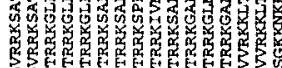

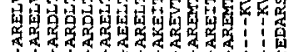

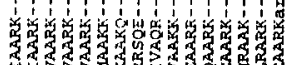

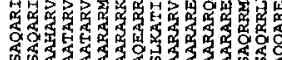

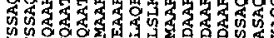

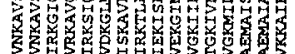

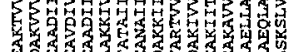

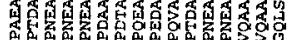

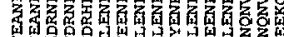

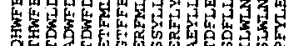

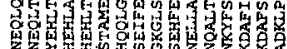

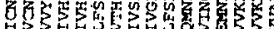

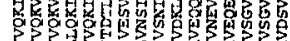

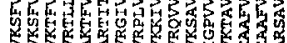

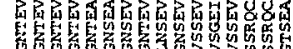

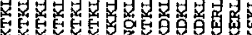

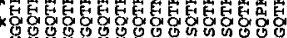

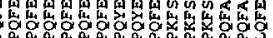

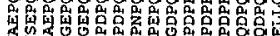

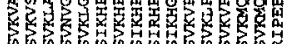

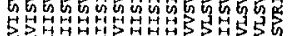

ᄀ3ำ

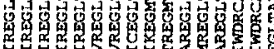

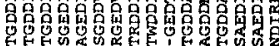

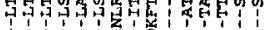

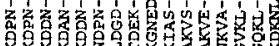

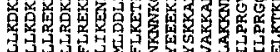

79

0.

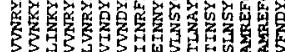

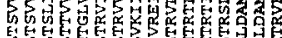

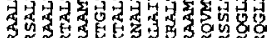

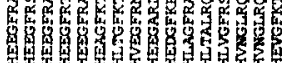

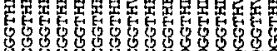

H.

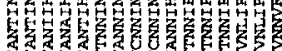

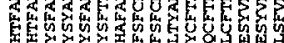

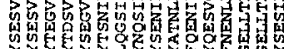

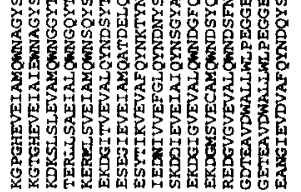

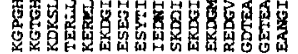

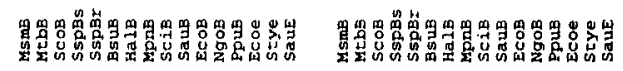




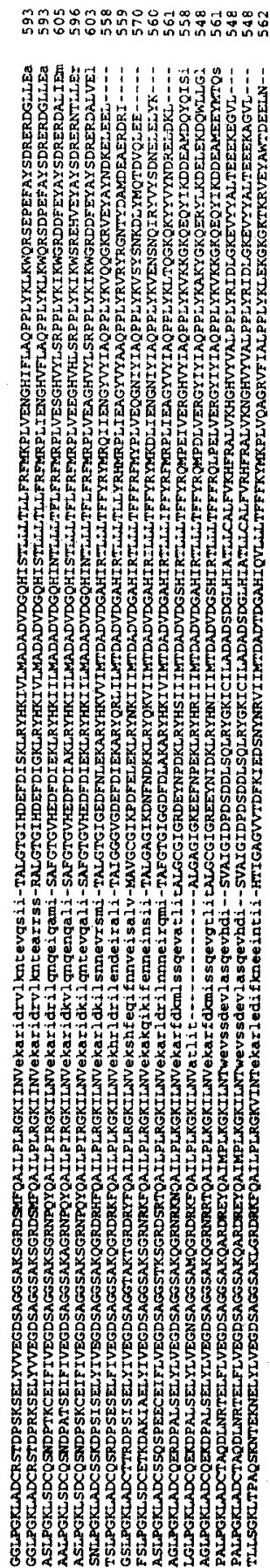

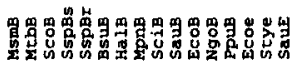
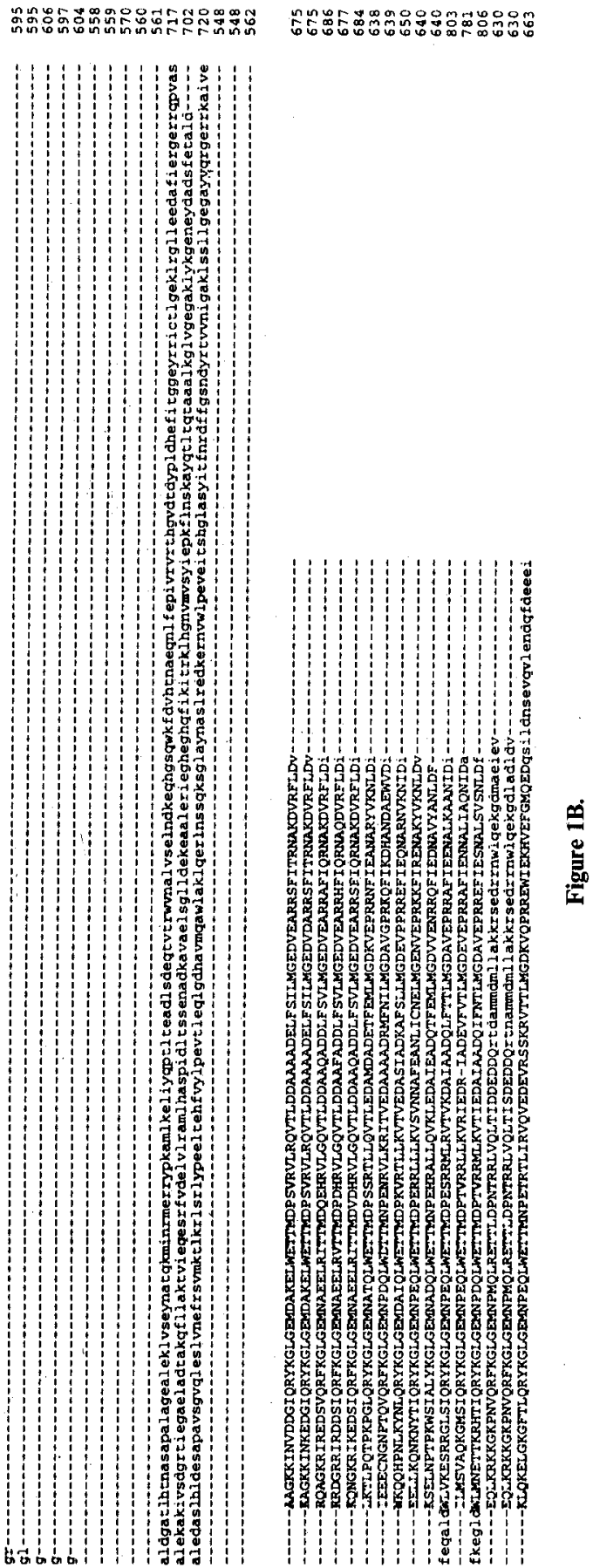

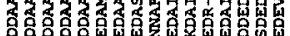

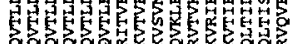

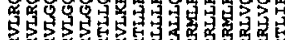

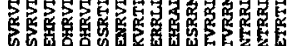

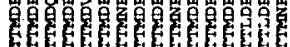

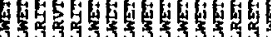

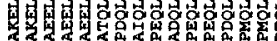

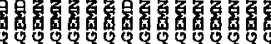
3 s.

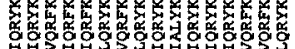

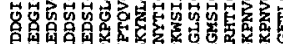

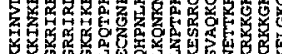

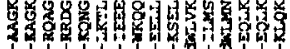

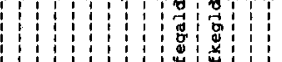




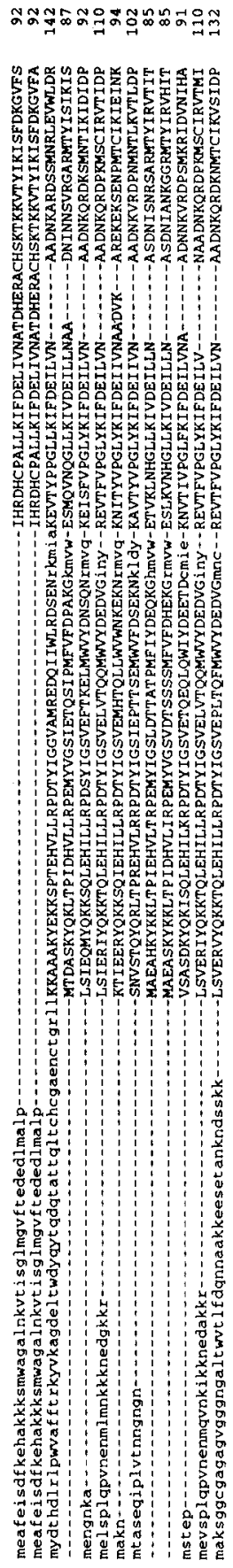

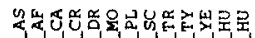

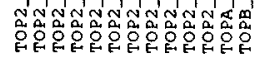

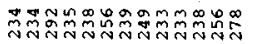

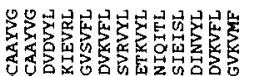

a

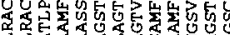

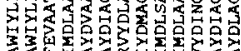

(1)

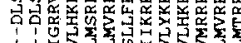

语

\&d

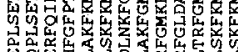

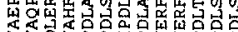

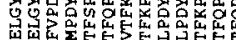

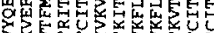

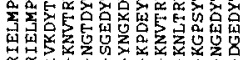

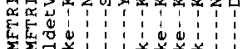

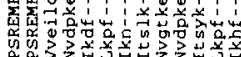

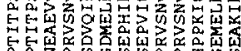

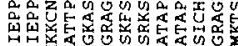

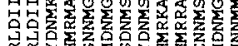

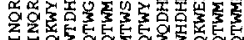

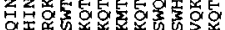

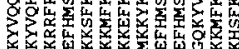

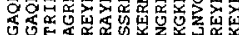

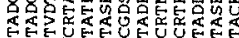

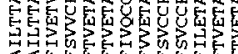

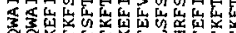

品

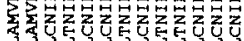

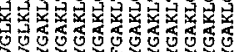

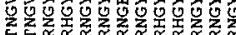

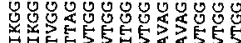

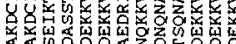

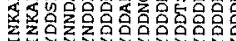

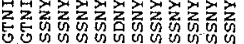

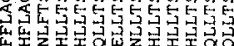

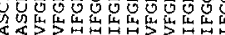

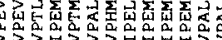

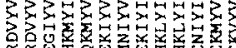

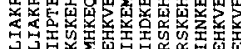

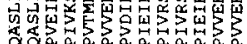

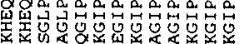

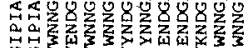

od

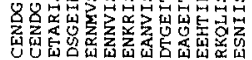

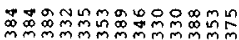

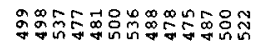

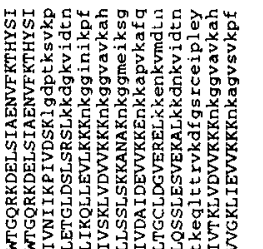

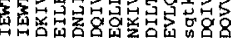

-

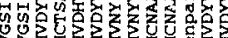

$\begin{array}{ll} & \\ 3 & \\ 3 & \end{array}$

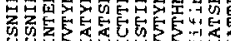

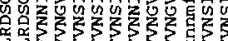

牙的

新和

原5

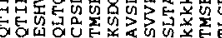

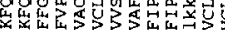

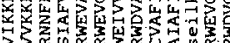

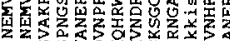

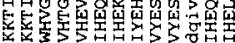

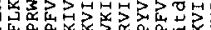

F5

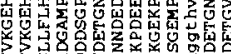

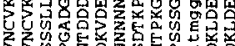

资

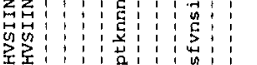

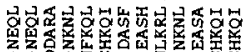

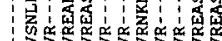

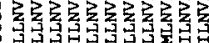

证

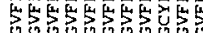

证

年

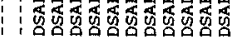

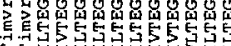

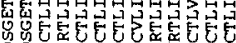

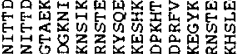

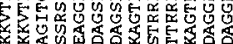

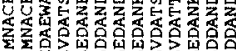

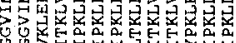

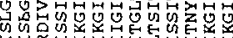

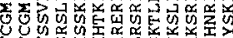

은

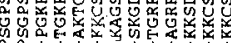

T.

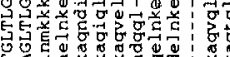

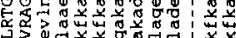

3)

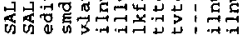

o

Jैy

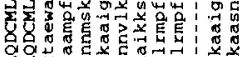

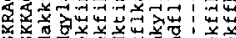

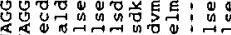

₹ ₹

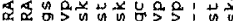

5⿹

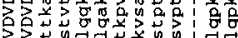

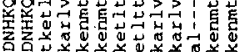

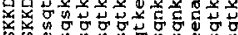

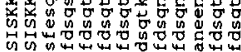

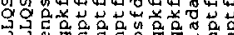

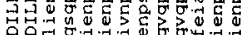

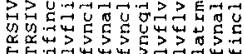

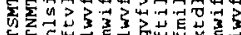

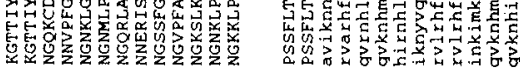

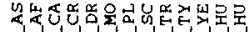

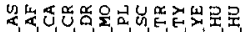

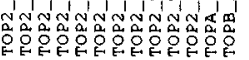

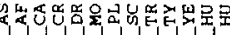

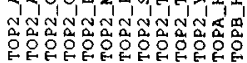



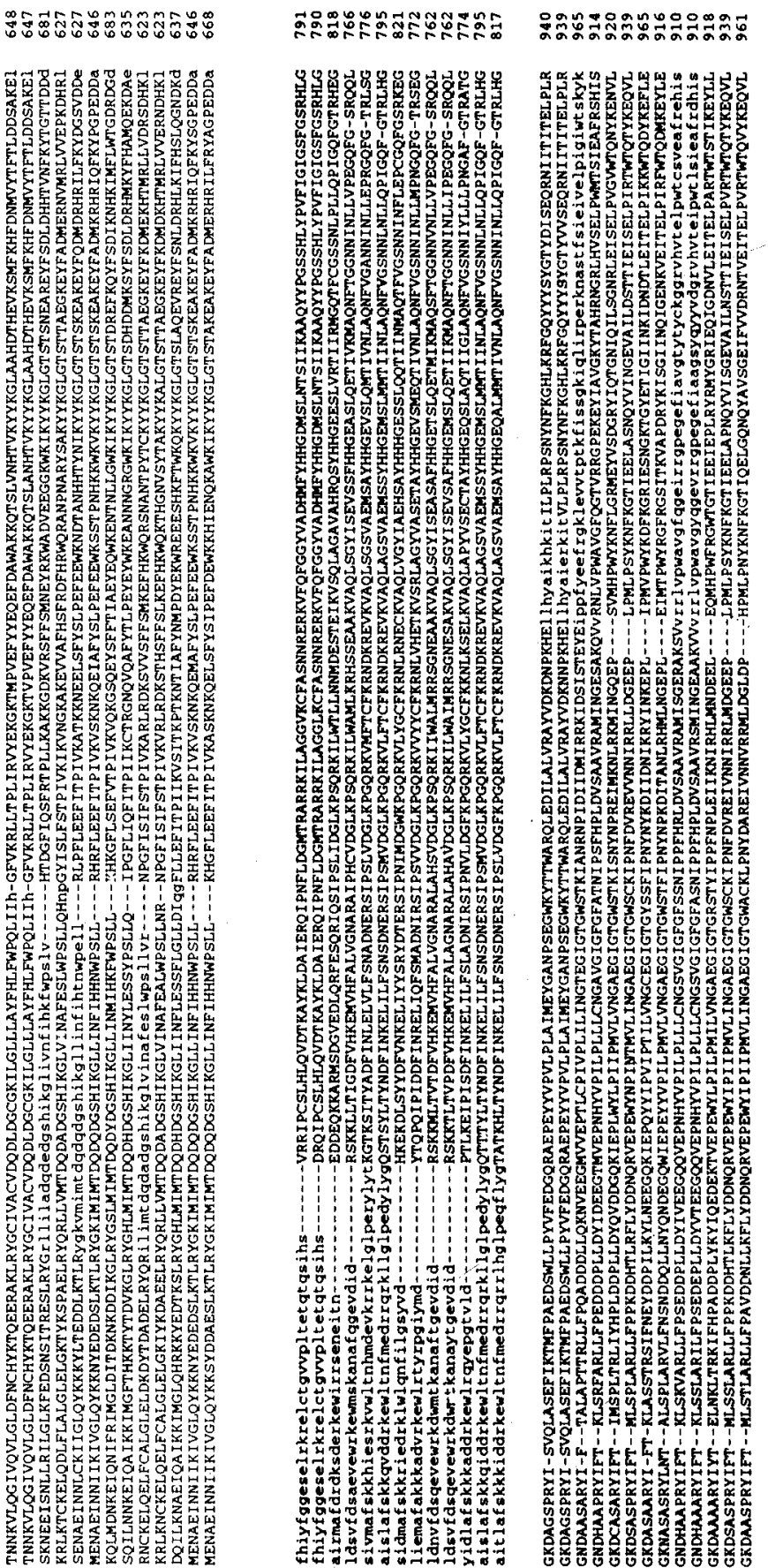

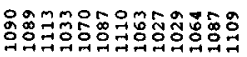

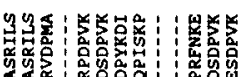

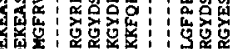

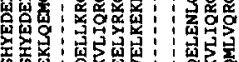

很离

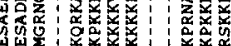

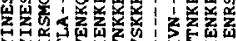

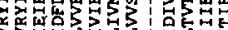

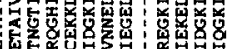

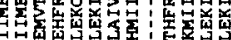

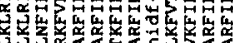

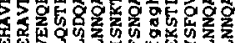

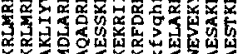

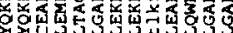

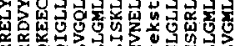

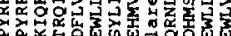

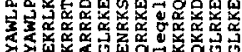

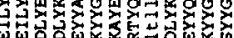

w

22

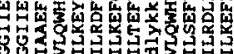

8 d

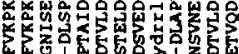

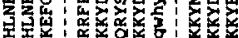

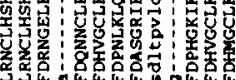

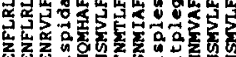

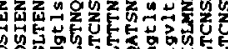

o.

56

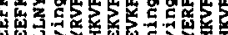

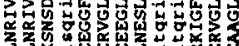

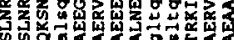

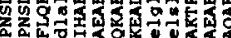

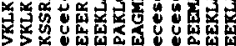

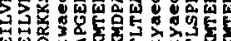

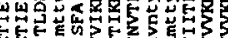

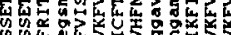

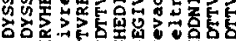

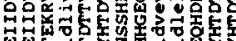

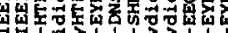

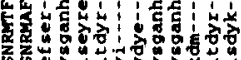
o.

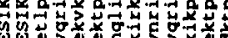
解

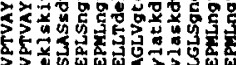

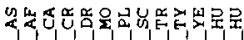

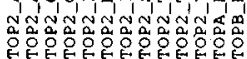




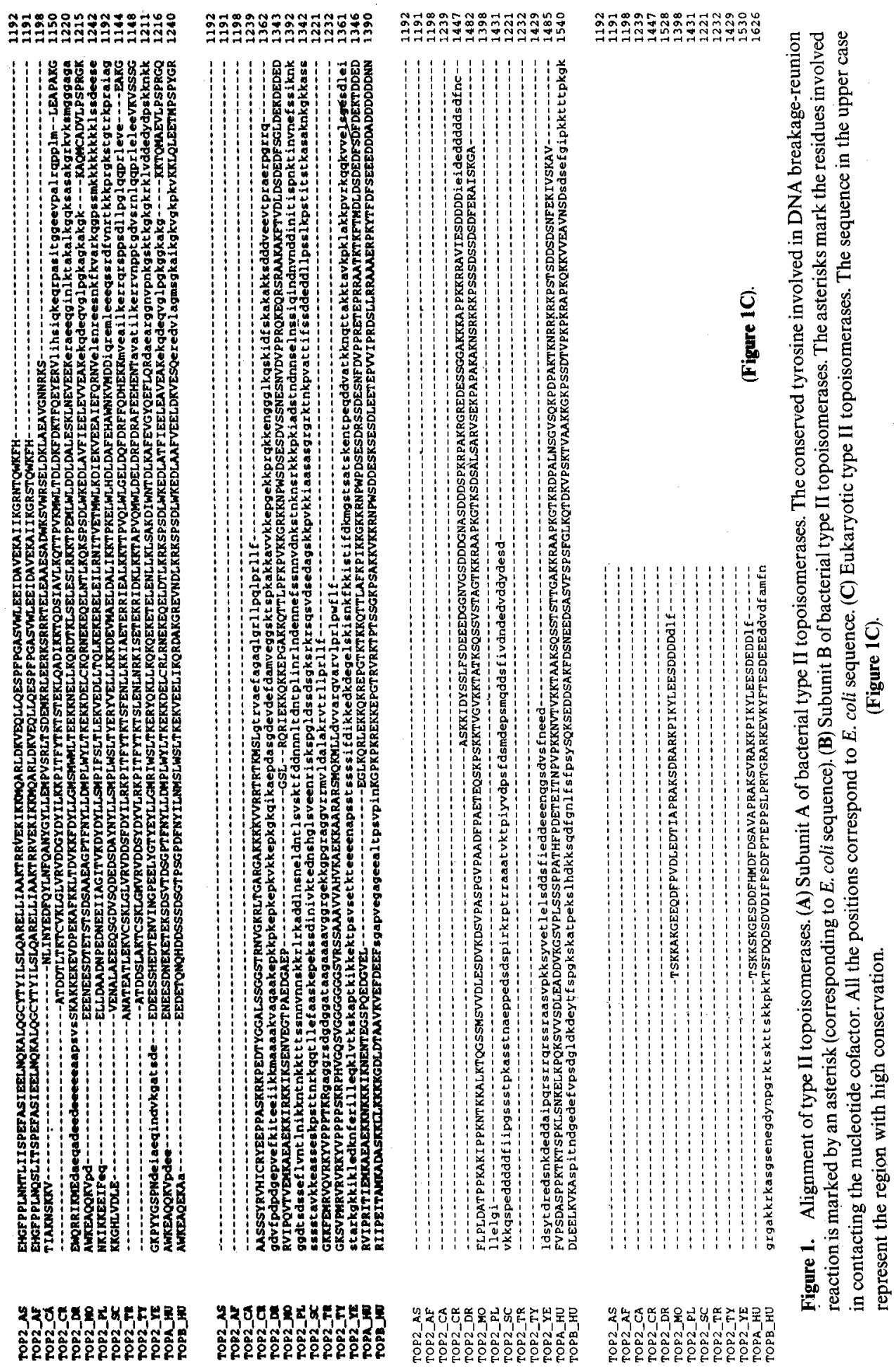


points, Tyr-5, Asn-46, Asp-73, Lys-103, Tyr-109, Gln-335 and Lys-337 are positionally conserved in all the members, except Spiroplasma citri wherein Lys-337 is replaced by Asn. The two important residues, Glu-42 and His-38 (Jackson and Maxwell 1994) implicated in ATPase activity of the subunit B of the $E$. coli enzyme are also present in all the other B sequences examined. A very significant difference is the absence of a long stretch of amino acids (158-163 amino acids) in GyrB proteins of Gram positive bacteria and Mycoplasma (figure 2B). The same stretch of amino acids is also absent in ParE sequences (B subunits of topoisomerase IV) of both Gram negative bacteria and $S$. aureus. It should also be noted here that gyrase and topoisomerase IV differ not only in their supercoiling ability but also in their potency of decatenation.

The subunits of bacterial type II topoisomerases were further analysed to understand the evolutionary relatedness. The unrooted trees are shown in figures 3 and 4 . The ParC proteins share a branch with GyrA of higher bacteria (figure 3). The ParE proteins are located closer to GyrB polypeptides of Gram negative bacteria (figure 4) in spite of sharing a common character (the absence of a long stretch of amino acids) with Gram positive bacteria and Mycoplasma (figure 2B). The B subunit of bacterial type II topoisomerases shows a distinct feature. All the GyrB proteins of Mycoplasma and Gram positive bacteria form a monophyletic group while other GyrB polypeptides and ParE sequences diverge into another group (figure 4). In case of A subunits, no such clear separation could be observed (figure 3 ).

The eukaryotic type II topoisomerases also show primary conservation to some extent. Although the sequences have diverged, functionally significant domains (like

MSmA

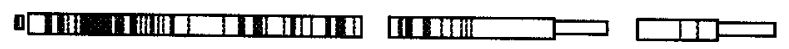

MitbA

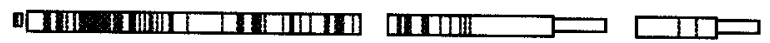

ScOA

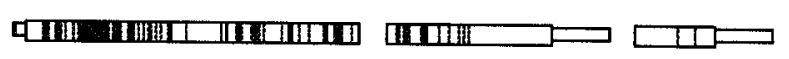

BSUA

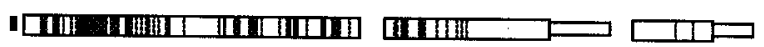

HalA

SauA

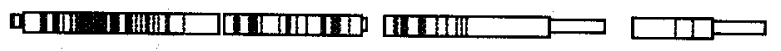

RprA

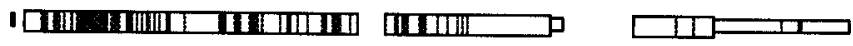

EcoA

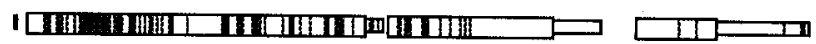

KpnA

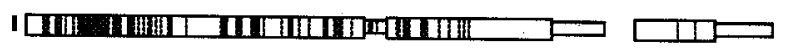

CajA

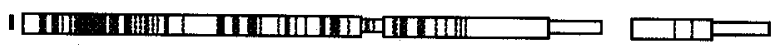

Sauc

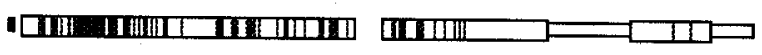

Styc

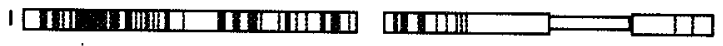

$\mathrm{ECOC}$

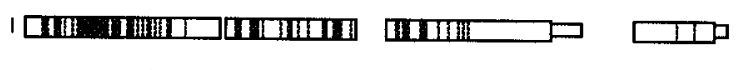

I [III IIIIII)

Figure 2A. 
$\mathbf{B}$

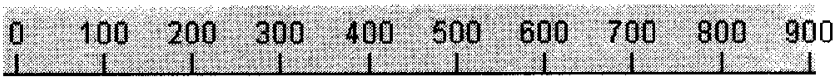

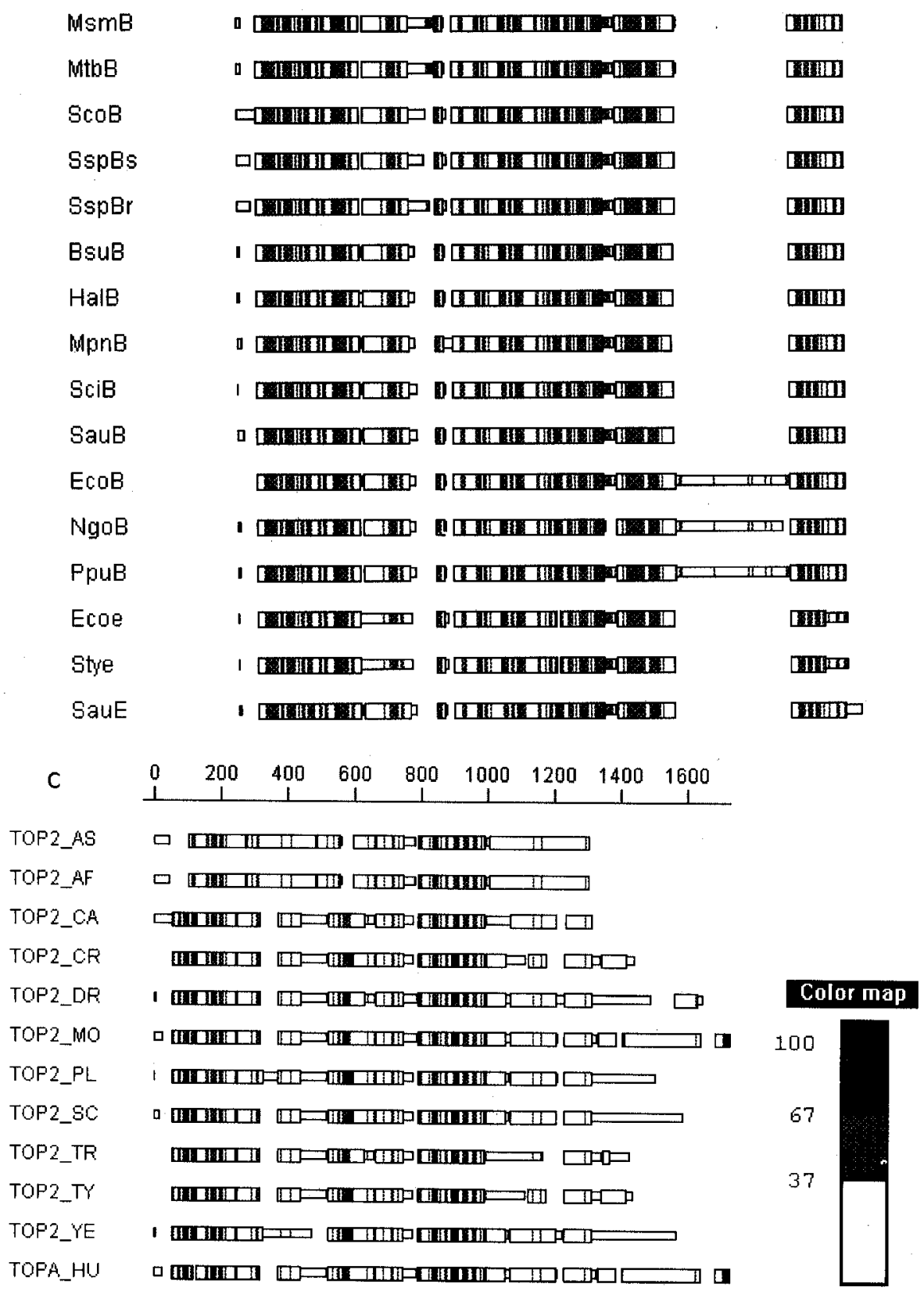

Figure 2. Schematic representation of multiple alignment derived from figure 1. The panels (A) (B) and (C) correspond to that of figure 1. The thick sequences correspond to blocks sharing significant similarity with the shaded regions representing the conserved sequence. The thin blocks represent the regions of the sequence not sharing statistically significant homology in the multiple alignment. 
(3)

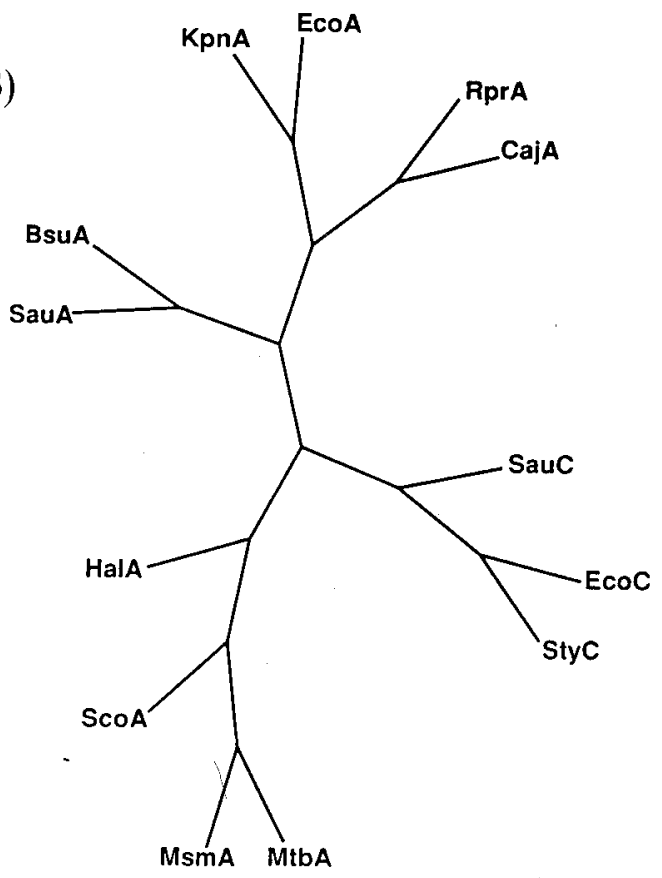

(4)

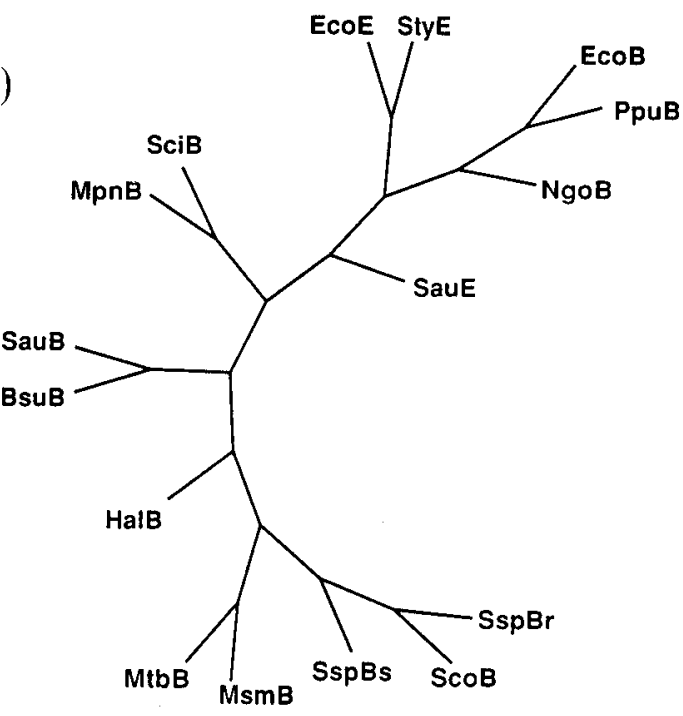

Figures 3 and 4. Evolutionary relationship among bacterial topoisomerase II subunits. Unrooted phylogenetic trees produced from the alignment of A subunits (3) in figure $1 \mathrm{~A}$ and B subunits $(4)$ in figure $1 \mathrm{~B}$, using DRAWTREE. 
ATPase and DNA breakage-reunion regions) have retained the residues important for the activities. The crystal structure of $92 \mathrm{kDa}$ domain of yeast DNA topoisomerase II at $2.7 \AA$ has been reported recently (Berger et al 1996). Whereas the N-terminal 409 amino acids constitute ATPase domain that shares homology with bacterial DNA gyrase subunit B ATPase region, this domain has been implicated in DNA cleavage and strand passage reactions required for the topological interconversion (Berger and Wang 1996). This

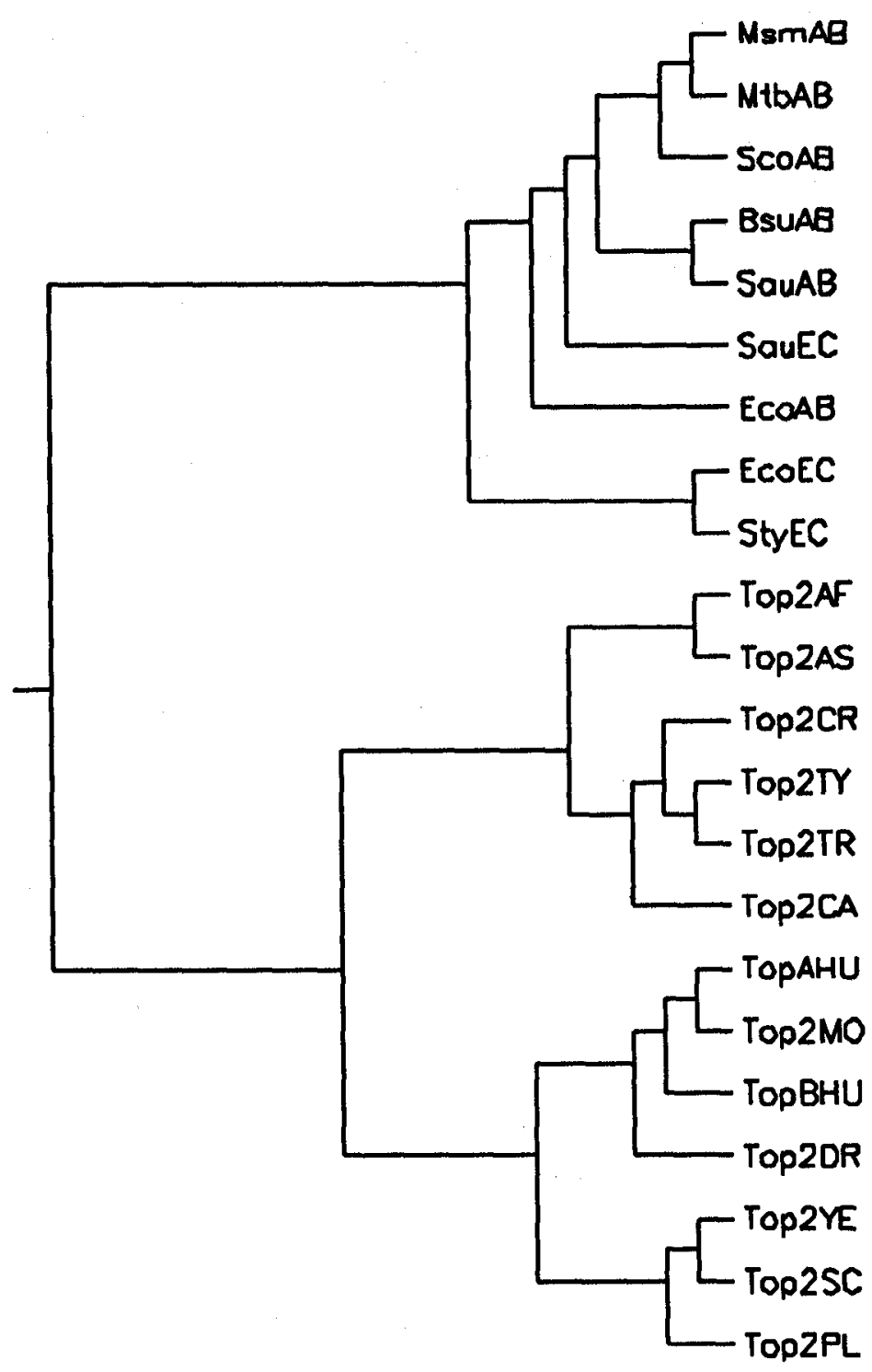

Figure 5. Phylogenetic tree of type II topoisomerases. The bacterial type II topoisomerase subunits were fused prior to the alignment. The abbreviations correspond to those given in the table 1, with $\mathrm{AB}$ representing fused sequences. 
domain contains GyrB and GyrA like sequences (Caron and Wang 1993). Also, this region shows high conservation among eukaryotic type II DNA topoisomerases (figures $1 \mathrm{C}$ and $2 \mathrm{C}$ ). Beyond this region, the amino acid sequences of eukaryotic type II DNA topoisomerases display less conservation. The cluster analysis of all type II topoisomerases, presented in figure 5, shows the monophyletic separation of bacterial sequences from the distinct diphyletic groups of eukaryotic enzymes.

\section{Acknowledgements}

We thank Shamala Prasad and Bindu D Paul for the assistance and C D Nager (Freidrich Miescher Institute, Basel) for introducing to MACAW. The sequence analysis was carried out at Bioinformatics Centre, Indian Institute of Science. The infrastructural facility was provided by Department of Biotechnology, New Delhi. KM is a recipient of Council of Scientifics and Industrial Research, New Delhi Senior Research Fellowship.

\section{References}

Adachi N, Miyaike M, Ikeda H and Kikuchi A 1992 Characterization of cDNA encoding the mouse DNA topoisomerase II that can complement the budding yeast top2 mutation; Nucleic Acids Res. 20 $5297-5303$

Adachi T, Mizuuchi M, Robinson E A, Apella E, O'Dea M H, Gellert M and Mizuuchi K 1987 DNA sequence of the $E$. coli gyr $B$ gene: application of a new sequencing strategy; Nucleic Acids Res. 15 771-784

Baylis S A, Dixon L K, Vydelingum S and Smith G L 1992 African Swine Fever virus encodes a gene with extensive homology to type II topoisomerase; J. Mol. Biol. 228 1003-1010

Berger J M, Gamblin S J, Harrison S C and Wang J C 1996 Structure and mechanism of DNA topoisomerase II; Nature (London) 379 225-232

Berger J M and Wang J C 1996 Recent developments in DNA topoisomerase II structure and mechanism; Curr. Op. Structural Biol. 6 84-90

Calcutt M J 1994 Improved shuttle vector for Haloferax volcanii including a dual-resistance plasmid; Gene $146117-121$

Caron P R and Wang J C 1993 DNA topoisomerases as target of therapeutics: A structural overview; in Molecular biology of DNA topoisomerases (Proceedings of International Symposium on DNA Topoisomerases in Chemotherapy, Nagoya, Japan) (Nov. 18-20, 1991) (New York: CRC press) pp 1-21

Colman S D, Hu P C and Bott K F 1990 Mycoplasma pneumoniae DNA gyrase genes; Mol. Microbiol. 4 1129-1134

Corpet J 1988 Multiple sequence alignment with heirarchical clustering; Nucleic Acids Res. 16 10881-10890

Dimri G P and Das H 1990 Cloning and sequence analysis of gyrA gene of Klebsiella pneumoniae; Nucleic Acids Res. 18 151-156

Felsenstein J 1989 PHYLIP-phylogeny inference package (version 3.2); Cladistics 5 164-166

Ferrero L, Cameron B, Manse B, Lagneaux D, Crouzet J, Famechon A and Blanche F 1994 Cloning and primary structure analysis of Staphylococcus aureus DNA topoisomerase IV: a primary target of fluoroquinolones; Mol. Microbiol. 13 641-653

Fragoso S P and Goldenberg S 1992 Cloning and characterization of the gene encoding Trypanosoma cruzi DNA topoisomerase II; Mol. Biochem. Parasitol. 55 127-134

Garcia-Beato R, Freije J M, Lopez-Otin C, Blasco R, Vinuela E and Salas M L 1992 A gene homologous to topoisomerase II in African swine fever virus; Virology 188 938-947

Gellert M, Mizuuchi K, O'Dea M H and Nash H 1976 DNA gyrase: an enzyme that introduces superhelical turns into DNA; Proc. Natl. Acad. Sci. USA 73 3872-3875

Giaever F, Lynn R, Goto T and Wang J C 1986 The complete nucleotide sequence of the structural gene TOP2 of yeast DNA topoisomerase II; J. Biol. Chem. 261 12448-12454 
Holmes M L and Dyall-Smith M L 1991 Mutations in DNA gyrase result in novobiocin resistance in halophilic archaebacteria; J. Bacteriol. 173 642-648

Holmes M L and Dyall-Smith M L 1994 Gene organization in the duaA-gyr A region of the Streptomyces coelicolor chromosome; Gene 146 117-121

Jackson A P and Maxwell A 1994 Identifying the catalytic residue of the ATPase reaction of DNA gyrase; Proc. Natl. Acad. Sci. USA $9011232-11236$

Jenkins J R, Ayton P, Jones T, Davies S L, Simmons D L, Harris A L, Sheer D and Hickson I D 1992 Isolation of cDNA clones encoding the $\beta$ isozyme of human DNA topoisomerase II and localization of the gene to chromosome; Nucleic Acids Res. 20 5587-5592

Kato J-I, Nishimura Y, Imamura R, Niki H, Iraga S and Suzuki H 1990 New topoisomerase essential for chromosome segregation in E. coli; Cell 63 393-404

Lawrence C E, Altschul S F, Boguski M S, Liu J S, Neuwald A F and Wootton J C 1993 Detecting subtle sequence signals: a Gibbs sampling strategy for multiple alignment; Science 62 208-214

Luttinger A L, Springer A L and Schmid M B 1991 A cluster of genes that affects nucleoid segregation in Salmonella typhimurium; New Biol. 3 687-697

Madhusudan K and Nagaraja V 1995 Mycobacterium smegmatis DNA gyrase: cloning and overexpression in Escherichia coli; Microbiology 140 3029-3037

Madhusudan K, Ramesh V and Nagaraja V 1994 Molecular cloning of gyr $A$ and gyr $B$ genes of $M y c o b a c-$ terium tuberculosis: analysis of nucleotide sequence; Biochem. Mol. Biol. Int. 33 651--660

Margerrison E E C, Hopewell R and Fisher L M 1992 Nucleotide sequence of the Staphylococcus aureus gyrB-gyrA locus encoding the DNA gyrase A and B proteins; J. Bacteriol. 174 1596-1603

Maxwell A and Gellert M 1986 Mechanistic aspects of DNA topoisomerses; Adv. Prot. Chem. 38 69-107

Moriya S, Ogasawara N and Yoshikawa $\mathrm{H} 1985$ Structure and function of the region of the replication origin of the Bacillus subtilis chromosome. III: nucleotide sequence of some 10,000 base pairs in the origin region; Nucleic Acids Res. 13 2251-2265

Parales R E and Harwood C S 1990 Nucleotide sequence of the gyrB gene of Pseudomonas putida; Nucleic Acids Res. 185880

Pasion S G, Hines J C, Aebersold R and Ray D S 1992 Molecular cloning and expression of the genes encoding kinetoplast-associated type II topoisomerase of Crithidia fasciculata; Mol. Biochem. Parasitol. 50 57-68

Reece R J and Maxwell A 1991 DNA gyrase: structure and function; CRC Rev. Biochem. Mol. Biol. 26 335-375

Ridley R G and Kilbey B J 1994 The gene encoding topoisomerase II from Plasmodium falciparum; Nucleic Acids Res. 22 2547-2551

Schuler G D, Altshul S F and Lipman D J 1991 A workbench for multiple alignment construction and analysis; Proteins 9 180-190

Springer A L and Schmid M B 1991 Molecular characterization of Salmonella typhimurium parE gene; Nucleic Acids Res. 21 1805-1809

Stein D C, Danaher R J and Cook T M 1991 Characterization of gyrB mutation responsible for low-level Nalidixic acid resistance in Neisseria gonorrhoeae; Antimicrob Agents Chemother. 35 622-626

Ştrauss P R and Wang J C 1990 The TOP2 gene of Trypanosoma brucei: a single copy gene that shares extensive homology with other TOP2 genes encodng eukaryotic DNA topoisomerase II; Mol. Biochem. Parasitol. 38 141-150

Swanberg S L and Wang J C 1987 Cloning and sequencing of the Escherichia coligyr A gene coding for the A subunit of DNA gyrase; $J$. Mol. Biol. 197 729-736

Takiff H E, Salazar L, Guerrero C, Philipp W, Huang W M, Kreisworth B, Cole S T, Jacobs W R Jr and Telenti A 1994 Cloning and nucleotide sequence of Mycobacterium tuberculosis gyr $A$ and gyr $B$ genes and detection of quinolone resistance mutations; Antimicrob Agents Chemother. 38 773-780

Thiara A S and Cundliffe E 1993 Expression and analysis of two gyr $B$ genes from the novobiocin producer, Streptomyces sphaeroides; Mol. Microbiol. 8 495-506

Tsai-Pflugfelder M, Liu L F, Liu A A, Tewey K M, Whang-Peng J, Knutsen T, Huebner K, Croce C M and Wang J C 1988 Cloning and sequencing of cDNA encoding human topoisomerase II and localisation of the gene to chromosome; Proc. Natl. Acad. Sci. USA 85 7177-7181

Uemura T, Morikawa K and Yanagida M 1986 The nucleotide sequence of the fission yeast DNA topoisomerase II gene: structural and functional relationships to other topoisomerases; EMBO J. $52355-2361$

Wallis J W, Chrebet G, Brodsky G, Rolfe M and Rothstein R 1989 A hyper-recombination mutation is $S$. cerevisiae identifies a novel eukaryotic topoisomerase; Cell $58409-419$ 
Wang J C 1985 DNA topoisomerases; Annu. Rev. Biochem. 54 665-697

Wang Y, Huang W M and Taylor D E 1993 Cloning and nucleotide sequence of the Campylobacter jejuni gyr $A$ gene and characterization of quinolone resistance mutations; Antimicrob Agents Chemother. 37 457-463

Wigley D B, Davies G J, Dodson E J, Maxwell A and Dodson G 1991 Crystal structure of the N-terminal domain of the DNA gyrase B protein; Nature (London) 351 624-629

Wilson R, Ainscough R, Anderson K et al $19942.2 \mathrm{Mb}$ of contiguous nucleotide from chromosome III of $C$. elegans; Nature (London) 368 32-38

Wood D O and Waite R T 1994 Sequence analysis of the Ricketsia prowazekii gyr A gene; Gene 151 191-196

WykoffE, Natalie D, Nolan J M, Lee M and Hsieh T-S 1989 Structure of the Drosophila DNA topoisomerase II gene: nucleotide sequence and homology among topoisomerases II; J. Mol. Biol. 205 1-13

Yamagishi J, Yoshida H, Yamayoshi M and Nakamura S 1986 Nalidixic acid-resistant mutations of the gyr $B$ gene of Escherichia coli; Mol. Gen. Genet. 204 367-373

Corresponding editor: VIDYANAND NANJUNDIAH 